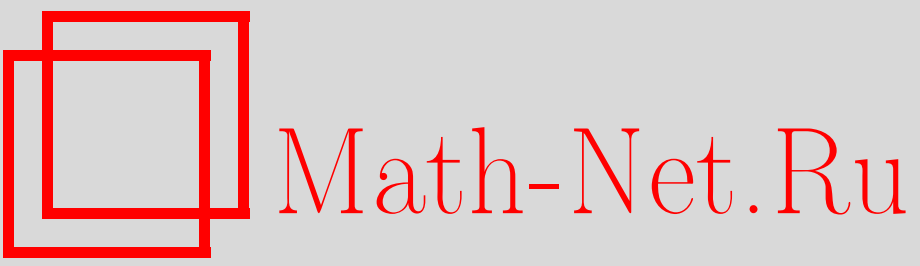

Д. Карраско-Оливера, Р. Мецгер, С. Моралес, Логарифмическое расширение, энтропия и размерность для многозначных отображений, Итоги науки и техн. Сер. Соврем. мат. и ее прил. Темат. обз., 2020, том 178, 31-40

DOI: https://doi.org/10.36535/0233-6723-2020-178-31-40

Использование Общероссийского математического портала Math-Net.Ru подразумевает, что вы прочитали и согласны с пользовательским соглашением

http://www.mathnet.ru/rus/agreement

Параметры загрузки:

IP : 54.196 .121 .252

26 апреля 2023 г., 13:27:45 


\title{
ЛОГАРИФМИЧЕСКОЕ РАСШИРЕНИЕ, ЭНТРОПИЯ И РАЗМЕРНОСТЬ ДЛЯ МНОГОЗНАЧНЫХ ОТОБРАЖЕНИЙ
}

\author{
(С) 2020 г. Д. КАРРАСКО-ОЛИВЕРА, Р. МЕЦГЕР, С. А. МОРАЛЕС
}

\begin{abstract}
АннотАция. Получена нижняя грань для энтропии (необязательно инвариантной) вероятностной борелевской меры относительно полунепрерывного сверху многозначного отображения как произведение нижней размерности меры и логарифмической скорости расширения отображения. Данная работа обобщает известный результат в однозначном случае, сохраняющем меру.
\end{abstract}

Ключевъе слова: логарифмическое расширение, метрическая энтропия, размерность.

\section{LOGARITHMIC EXPANSION, ENTROPY, AND DIMENSION FOR SET-VALUED MAPS}

\author{
(c) 2020 D. CARRASCO-OLIVERA, R. METZGER, C. A. MORALES
}

\begin{abstract}
We obtain a lower bound for the entropy of a (not necessarily invariant) Borel probability measure with respect to an upper semicontinuous set-valued map as the product of the lower dimension of the measure and the logarithmic expansion rate. This is a generalization of the well-known measurepreserving single-valued case.
\end{abstract}

Keywords and phrases: logarithm expansion, metric entropy, dimension.

AMS Subject Classification: 37D40, 54C60

1. Введение. Анализ специфических экономических, биологических и химических моделей часто сталкивается с задачей поиска положений равновесия для некоторых динамических систем. Эти положения соответствуют решениям, которые могут вести себя случайно либо не в соответствии с хаотичностью системы, что порождает проблему анализа хаотичности системы. Одной из наиболее популярных величин, характеризующих хаос при условии однозначности, является энтропия. Это понятие имеет долгую историю, начиная с его введения в термодинамике Р. Клаузиусом в середине XIX в. (см. [18,19]. Дальнейшее развитие этого численного инварианта восходит к статистической и квантовой механике (см. [13,37]) и теории информации (см. $[4,29,33])$. Также известны метрическая энтропия Колмогорова-Синая (см. [2,3]) и топологическая энтропия Адлера-Конхейма-МакЭндрю (см. [5]). Читатель может найти ссылки и исторические заметки в $[21,25,34]$. Совсем недавно авторы рассмотрели топологическую энтропию для многозначных отображений (см. [16]).

Работа Д. Карраско-Оливера выполнена при поддержке проекта FONDECYT 1181061, CONICYT (Чили).

Работа Р. Мецгера выполнена при поддержке проекта Pos-Doutorado Verano 2015, IMPA, Рио-де-Жанейро, Бразилия.

Работа С. А. Моралеса выполнена при поддержке проектов CNPq-Brazil и MATHAMSUB 15 MATH05ERGOPTIM.. 
В данной работе продолжается изучение метрической энтропии для многозначных отображений. Проанализирована задача поиска нижних границ метрической энтропии в указанных условиях. Начало этому исследованию положил результат Р. Манэ (см. [28]) о том, что компактные метрические пространства, связанные расширенными гомеоморфизмами, имеют конечную топологическую размерность. Позже А. Фати (см. [22]) обнаружил существование нижней границы для топологической энтропии в виде произведения размерности Хаусдорфа и логарифмического расширения гомеоморфизма. Дай, Жу и Женг (см. [20]) расширили результат Фати до метрической энтропии Колмогорова-Синая. Цель данной работы-расширить полученные границы в контексте многозначных отображений. В работе будет получена нижняя граница для метрической энтропии полунепрерывного сверху многозначного отображения как произведения нижней размерности меры и логарифмической скорости расширения отображения (см. $[24,32])$. В частности, скорость расширения и размерность пространственного окружения можно использовать для определения хаотичности данной многозначной модели.

Рассмотрим метрическое пространство $X$. Обозначим через $B[a, r]$ замкнутый шар с центром $a$ и радиусом $r$. Для подмножеств $A, B \subset X$, определим расстолние между $A$ и $B$ формулой

$$
\operatorname{dist}(A, B)=\inf \{d(a, b): a \in A, b \in B\} .
$$

Обозначим через $2^{X}$ множество всех подмножеств $X$. Под многозначным отображсением $X$ понимается отображение $f: X \rightarrow 2^{X}$. Считается, что многозначное отображение $f$ пространства $X$ является замкнутозначным, если $f(x)$ замкнуто при каждом $x \in X$, и однозначным, если $\operatorname{card}(f(x))=1$ при всех $x \in X$, где card обозначает мощность. Существует очевидное соответствие между однозначными отображениями $f: X \rightarrow 2^{X}$ и отображениями $f: X \rightarrow X$. Композиция $f \circ g$ многозначных отображений $f, g: X \rightarrow 2^{X}$ определяется с помощью формулы

$$
(f \circ g)(x)=\bigcup_{y \in g(x)} f(y) \quad \forall x \in X .
$$

Введем тождественное отображение $f^{0}=I d$ и индуктивно определим $f^{k}=f^{k-1} \circ f$ для каждого $k \in \mathbb{N}^{+}$. Далее предположим, что каждое многозначное отображение $f$ является строгим, т.е. $f(x) \neq \emptyset$ для каждого $x \in X$ (подробнее о многозначном анализе и многозначной динамики см. $[6-9,15,27,31,35,36])$.

Определим константу расширения $E C(f)$ многозначного отображения $f$ пространства $X$ как такое наибольшее значение $\rho \geqslant 0$, что для каждого $0<\rho^{*}<\rho$ существует такое $\delta>0$, что $\operatorname{dist}(f(x), f(y)) \geqslant \rho^{*} d(x, y)$, если $x, y \in X$ удовлетворяют условию $d(x, y) \leqslant \delta$. Это определение расширяет соответствующее определение для однозначных отображений (см. [24]) до общих многозначных отображений. Каждое многозначное отображение $f$, удовлетворяющее условию $E C(f)>1$, является (положительно) расширяющим в смысле Уильямса (см. [39]). Однозначные отображения называют также отображениями, расширяющими малые расстояния (см. [30]).

Обобщим определение логарифмической скорости расширения с однозначного (например, [32]) до общего многозначного случая.

Определение 1.1. Логарифмическая скорость расширения многозначного отображения $f$ определяется следующим образом:

$$
\xi(f)=\sup _{k \geqslant 0} \frac{1}{k} \log E C\left(f^{k}\right) .
$$

Многозначное отображение $f$ пространства $X$ называется непрерывным сверху, если для каждого $x \in X$ и каждой окрестности $\mathcal{U}$ множества $f(x)$ существует такое $\eta>0$, что $f\left(x^{\prime}\right) \subset \mathcal{U}$ при каждом $x^{\prime} \in X$, удовлетворяющем условию $d\left(x, x^{\prime}\right)<\eta$. Это определение сводится к обычной классической непрерывности в случае однозначного отображения. Если $X$ является компактом, это определение эквивалентно следующему: для каждого $\epsilon>0$ существует такое $\eta>0$, что $f(x) \subset B(f(x), \epsilon)$ при любом $x^{\prime} \in X$, удовлетворяющем условию $d\left(x, x^{\prime}\right)<\eta$ (см. [8]). 
Любому $n \in \mathbb{N}^{+}$поставим в соответствие отображение $d_{n}: X \times X \rightarrow \mathbb{R}^{+}$, определенное по правилу

$d_{n}(x, y)=\inf \left\{\sup _{0 \leqslant i \leqslant n-1} d\left(x_{i}, y_{i}\right):\left(x_{i}\right)_{i=0}^{n}, \quad\left(y_{i}\right)_{i=0}^{n}\right.$ являются последовательностями,

удовлетворяющими условиям $x_{0}=x, y_{0}=y, x_{i+1} \in f\left(x_{i}\right), y_{i+1} \in f\left(y_{i}\right)$ при $\left.0 \leqslant i \leqslant n-1\right\}$.

Обозначение $d_{n}^{f}$ показывает зависимость от $f$.

Эти отображения являются метриками в случае однозначности, но лишь полуметриками в общем случае (см. [12,23] и пример 2.2 ниже).

Определим $\epsilon$-шары, центрированные в $x \in X$ относительно $d_{n}$ :

$$
B_{n}[x, \epsilon]=\left\{y \in X: d_{n}(x, y) \leqslant \epsilon\right\} ;
$$

в обозначении $B_{n}^{f}[x, \epsilon]$ указана зависимость от $f$.

Следуя $[1,14]$, авторы определили в [16] топологическую энтропию многозначного отображения $f$ пространства $X$ с помощью равенства

$$
h(f)=\lim _{\epsilon \rightarrow 0} \limsup _{n \rightarrow \infty} \frac{1}{n} \log r(n, \epsilon),
$$

где $r(n, \epsilon)$ - максимальная мощность $(n, \epsilon)$-порождающего множсества, т.е. множества $E$, удовлетворяющего условию

$$
X=\bigcup_{x \in E} B_{n}[x, \epsilon]
$$

Аналогичное понятие может быть введено с помощью замены $r(n, \epsilon)$ в $(2)$ на минимальную мощность $(n, \epsilon)$-разделенного множества $F$, удовлетворяющего условию $B_{n}[x, \epsilon] \cap F=\{x\}$ при всех $x \in F$ (см. [16]).

Введем похожий численный инвариант для меры Бореля, мотивированный (2) (см. [26]).

Пусть $\mu$ и $f$ обозначают меру Бореля и многозначное отображение пространства $X$ соответственно. Не предполагается, что $\mu$ является инвариантом для $f$ в смысле многозначности (см. [8]). Для любого $A \subset X$ и $\delta>0$ считаем, что $\mu(A) \leqslant \delta$, если $\mu(B) \leqslant \delta$ для любого борелевского подмножества $B \subset A$.

При заданном $n \in \mathbb{N}^{+}$и $\epsilon, \delta>0$ подмножество $E \subset X$ называется $(n, \epsilon, \delta)$-порождающим для $\mu$ относительно $f$, если

$$
\mu\left(X \backslash \bigcup_{x \in E} B_{n}[x, \epsilon]\right) \leqslant \delta
$$

(см. [17, с. 488]). Введем величину

$$
r(n, \epsilon, \delta)=\min \{\operatorname{card}(E): E \text { является }(n, \epsilon, \delta) \text {-порождающим }\} .
$$

Будем писать $r(f, \mu, n, \epsilon, \delta)$, чтобы подчеркнуть зависимость от $f$ и $\mu$. Возможно, что $r(n, \epsilon, \delta)=$ $\infty$; ниже в этих случаях полагаем, что $\log \infty=\infty$. Определим

$$
h(f, \mu, \epsilon, \delta)=\limsup _{n \rightarrow \infty} \frac{\log r(n, \epsilon, \delta)}{n} .
$$

Очевидно, $r(n, \epsilon, \delta) \leqslant r\left(n, \epsilon^{\prime}, \delta\right)$, а потому и $h(f, \mu, \epsilon, \delta) \leqslant h\left(f, \mu, \epsilon^{\prime}, \delta\right)$ при $0<\epsilon^{\prime} \leqslant \epsilon$. Отсюда следует, что существует предел

$$
h(f, \mu, \delta)=\lim _{\epsilon \rightarrow 0} h(f, \mu, \epsilon, \delta)=\sup _{\epsilon>0} h(f, \mu, \epsilon, \delta) .
$$

Подобным образом $r\left(n, \epsilon, \delta^{\prime}\right) \geqslant r(n, \epsilon, \delta)$, когда $\delta^{\prime} \leqslant \delta$, поэтому также существует предел

$$
\lim _{\delta \rightarrow 0} h(f, \mu, \delta)=\sup _{\delta>0} h(f, \mu, \delta) .
$$

Используя эти замечания, можно сформулировать следующие определения. 
Определение 1.2. Метрическая энтропия $\mu$ относительно $f$ определяется формулой

$$
h(f, \mu)=\lim _{\delta \rightarrow 0} h(f, \mu, \delta) .
$$

Определение 1.3. Будем говорить, что $\mu$ имеет ниюнюю размерность $\underline{d}(\mu) \in \mathbb{R}^{+}$, если

$$
\liminf _{r \rightarrow 0} \frac{\log \mu(B[x, r])}{\log r}=\underline{d}(\mu) \quad \text { равномерно по } x \in X .
$$

По сравнению с обычной нижней размерностью это определение является более сильным, требующим лишь существование предела величины $\mu$ при почти всех $x$ (см. $[10,11])$.

Используя эти определения, сформулируем результат данной работы.

Теорема 1.4. Для любого полунепрерывного сверху замкнутозначного отображения $f: X \rightarrow$ $2^{X}$ и любой вероятностой борелевской меры $\mu$ с ниюсней размерностъю $\underline{d}(\mu)$ компактного метрического пространства $X$ выполнено неравенство

$$
h(f, \mu) \geqslant \underline{d}(\mu) \cdot \xi(f) .
$$

Повторим, что от меры $\mu$ не требуется быть инвариантной. Конечно, интересен случай, когда обе величины $\underline{d}(\mu)$ и $\xi(f)$ положительны. Нетривиальный (т.е. не однозначный) пример, удовлетворяющий этим гипотезам, рассмотрен ниже (см. пример 2.1).

\section{2. Примеры.}

Пример 2.1. Равномерно непрерывное замкнутозначное отображение $f$, не являющееся однозначным, для которого $\xi(f)>0$, и такая мера Бореля $\mu$ компактного интервала $[0,1]$, что $\underline{d}(\mu)>0$ :

Наделим единичный интервал $[0,1]$ евклидовой метрикой. Определим многозначное отображение $f$ интервала $[0,1]$ следующим образом:

$$
f(x)= \begin{cases}\{2 x\}, & \text { если } 0 \leqslant x<\frac{1}{2}, \\ \{0,1\}, & \text { если } x=\frac{1}{2}, \\ \{2 x-1\}, & \text { если } \frac{1}{2}<x \leqslant 1 .\end{cases}
$$

Следовательно, $\xi(f)=\log 2$. Принимая $\mu$ в качестве меры Лебега, имеем $\underline{d}(\mu)=1$.

Пример 2.2. Многозначное отображение, для которого ассоциированные полуметрики $d_{n}$ в (1) не являются метриками. Этот пример важен, так как он показывает, что энтропия, которая рассматривается в данной работе для многозначных отображений, имеет некоторые отличия от стандартной однозначной энтропии.

Для каждого $k \in \mathbb{N}^{+}$существует многозначное отображение $f$ в сферу $S^{k}$, для которого $d_{n}$ не является метрикой при $n \geqslant 2$.

Доказательство. Выберем три различные точки $a, b, c \in S^{k}$ и три подмножества $A, B, C \subset S^{k}$, удовлетворяющие следующим трём гипотезам:

(i) $\operatorname{dist}(A, B)=\operatorname{dist}(B, C)=0$;

(ii) $\max (d(a, b), d(b, c))<\frac{1}{2} \operatorname{dist}(A, C)$;

(iii) $\{a, b, c\} \cap(A \cup B \cup C)=0$.

Так как $a \neq b \neq c \neq a$, то корректно определено следующее многозначное отображение $f$ сферы $S^{k}$ :

$$
f(x)= \begin{cases}A, & \text { если } x=a, \\ B, & \text { если } x=b, \\ C, & \text { если } x=c, \\ \{x\}, & \text { если } x \notin\{a, b, c\} .\end{cases}
$$

Из первых двух гипотез следует, что $d_{2}$ не является метрикой для этого $f$. Используя третью гипотезу, получаем $d_{n}=d_{2}$ для каждого $n \geqslant 2$. Тогда $d_{n}$ не является метрикой при любом $n \geqslant 2$. 
Сформулируем некоторые свойства энтропии, которые будут полезны для вычислительных целей. Как обычно, пишем $f \leqslant g$ для многозначных отображений $f$ и $g$, если $f(x) \subset g(x)$ при всех $x \in X$. Любая мера Бореля $\mu$ обладает следующими свойствами:

(Н1) если $f \leqslant g$, то $h(f, \mu) \leqslant h(g, \mu)$ (сохранение порядка);

(H2) $h(f, \mu) \leqslant h\left(f^{k}, \mu\right) \leqslant k \cdot h(f, \mu)$ для всех $k \in \mathbb{N}^{+}$(энтропия степени);

(Н3) $h(f, \mu) \leqslant h(f)$ (полувариационный принцип).

Второе неравенство в (Н2) будет доказано в лемме 3.2. Оставшиеся неравенства легко следуют из определений.

Известно, что тождество $I(x)=x$ на любом компактном метрическом пространстве имеет нулевую топологическую энтропию. Следующий пример расширяет этот факт до многозначного контекста.

Пример 2.3. Пусть $f$-многозначное отображение компактного метрического пространства $X$. Если существует $k \in \mathbb{N}^{+}$, удовлетворяющее условию $x \in f^{k}(x)$ для всех $x \in X$, то $h(f, \mu)=0$ для любой меры Бореля $\mu$.

Доказательство. Согласно (Н2) можно предположить, что $k=1$. Докажем, что $d_{n}^{f}=d$ для любого $n \in \mathbb{N}^{+}$. Неравенство $d_{n}^{f} \geqslant d$ легко следует из определения. Теперь выберем $x, y \in X$ и $n \in \mathbb{N}^{+}$и определим последовательности $\left(x_{i}\right)_{i=0}^{n}$ и $\left(y_{i}\right)_{i=0}^{n}$ формулами $x_{i}=x$ и $y_{i}=y$ для всех $0 \leqslant i \leqslant n$. Очевидно, эти последовательности удовлетворяют условиям $x_{0}=x, y_{0}=y$, $x_{i+1}=x \in f(x)=f\left(x_{i}\right)$ и $y_{i+1}=y \in f(y)=f\left(y_{i}\right)$ для всех $0 \leqslant i \leqslant n-1$. Следовательно,

$$
d_{n}^{f}(x, y) \leqslant \sup _{0 \leqslant i \leqslant n-1} d\left(x_{i}, y_{i}\right)=\sup _{0 \leqslant i \leqslant n-1} d(x, y)=d(x, y) .
$$

Заключаем, что $d_{n}^{f} \leqslant d$ и, таким образом, $d_{n}^{f}=d$ для любого $n \in \mathbb{N}^{+}$. Значит, $r(f, \mu, n, \epsilon, \delta)$ не зависит от $n$ при любой мере Бореля $\mu$, и поэтому $h(f, \mu)=0$.

В следующем примере вычислена энтропия субдифференциальной евклидовой нормы $f(x)=$ $|x|$ пространства $\mathbb{R}$ (см. [31, с. 215]).

Пример 2.4. Определим многозначное отображение $\partial f$ интервала $[-1,1]$ следующим образом:

$$
\partial f(x)= \begin{cases}\{1\}, & \text { если }-1 \leqslant x<0, \\ {[-1,1],} & \text { если } x=0, \\ \{-1\}, & \text { если } 0<x \leqslant 1 .\end{cases}
$$

Оно удовлетворяет условию $h(\partial f, \mu)=0$ при любой мере Бореля $\mu$ интервала $[-1,1]$.

Доказательство. Сначала докажем, что величина $h(\partial f)$, определенная в (2), удовлетворяет условию $h(\partial f)=0$. Заметим, что из свойства сохранения порядка (Н1) следует неравенство $h(\partial f) \leqslant h(s)$, где $s$ - селектор $\partial f$, определенный следующим образом:

$$
s(x)= \begin{cases}1, & \text { если }-1 \leqslant x<0, \\ 0, & \text { если } x=0 \\ -1, & \text { если } 0<x \leqslant 1 .\end{cases}
$$

Пусть $E$ является $(1, \epsilon)$-порождающим множеством $s$. Задавая $\epsilon$ малым, можно обеспечить, чтобы $E$ имело точки по обе стороны от 0. Следовательно, $E^{\prime}=\{0\} \cup E$ является $(n, \epsilon)$-порождающим для каждого $n \in \mathbb{N}^{+}$. Из этого получаем $r(n, \epsilon) \leqslant r(1, \epsilon)+1$ для каждого $n \in \mathbb{N}^{+}$. Очевидно, $r(1, \epsilon)<\infty$, поэтому

$$
h(s) \leqslant \lim _{\epsilon \rightarrow 0} \limsup _{n \rightarrow \infty} \frac{1}{n} \log (r(1, \epsilon)+1)=0 .
$$

Так как $h(\partial f)=0$, получаем $h(\partial f, \mu)=0$ с помощью полувариационного принципа (Н3). 
3. Доказательство теоремы 1.4. Начнем с леммы, которая известна в однозначном случае.

Лемма 3.1. Для любого многозначного отображения $f$ метрического пространства $X$ име$e \mathcal{M}$

$$
B_{k n}^{f}[x, \epsilon] \subset B_{n}^{f^{k}}[x, \epsilon] \quad \forall x \in X, n \in \mathbb{N}^{+}, \epsilon>0 .
$$

Доказательство. Достаточно доказать, что

$$
d_{n}^{f^{k}} \leqslant d_{k n}^{f} \quad \forall n, k \in \mathbb{N}^{+} .
$$

Можно полагать, что $k \in \mathbb{N}^{+}$. Зафиксируем $n \in \mathbb{N}^{+}$и $x, y \in X$. Пусть дано $\gamma>0$. Существуют такие последовательности $\left(x_{i}\right)_{i=0}^{k n}$ и $\left(y_{i}\right)_{i=0}^{k n}$, что $x_{0}=x, y_{0}=y, x_{i+1} \in f\left(x_{i}\right), y_{i+1} \in f\left(y_{i}\right)$ и $d\left(x_{i}, y_{i}\right) \leqslant d_{k n}^{f}(x, y)+\gamma$ при $0 \leqslant i \leqslant k n-1$. Определим $\hat{x}_{i}=x_{k i}$ и $\hat{y}_{i}=y_{k i}$ для $0 \leqslant i \leqslant n$. Тогда результирующие последовательности $\left(\hat{x}_{i}\right)_{i=0}^{n}$ и $\left(\hat{y}_{i}\right)_{i=0}^{n}$ удовлетворяют соотношениям $\hat{x}_{0}=x$, $\hat{y}_{0}=y, \hat{x}_{i+1} \in f^{k}\left(\hat{x}_{i}\right), \hat{y}_{i+1} \in f^{k}\left(\hat{y}_{i}\right)$ и $d\left(\hat{x}_{i}, \hat{y}_{i}\right) \leqslant d_{k n}^{f}(x, y)+\gamma$ при $0 \leqslant i \leqslant n-1$. Тогда $d_{n}^{f^{k}}(x, y) \leqslant$ $d_{n}^{f}(x, y)+\gamma$. Так как значение $\gamma$ произвольно, получаем требуемый результат.

Далее сформулируем многозначную версию известного свойства метрической энтропии однозначного отображения.

Лемма 3.2. Если $f$-многозначное отображение метрического пространства, то

$$
h\left(f^{k}, \mu\right) \leqslant k \cdot h(f, \mu) \quad \forall K \in \mathbb{N}^{+}
$$

для любой меры Бореля $\mu$.

Доказательство. Пусть $n \in \mathbb{N}^{+}, \epsilon>0, \delta>0$ и $E-(k n, \epsilon, \delta)$-порождающее множество для $\mu$ относительно $f$. С помощью леммы 3.1 получаем

$$
\left(X \backslash \bigcup_{x \in E} B_{n}^{f^{k}}[x, \epsilon]\right) \subset\left(X \backslash \bigcup_{x \in E} B_{k n}^{f}[x, \epsilon]\right) ;
$$

следовательно, $E$ является $(n, \epsilon, \delta)$-порождающим для $\mu$ относительно $f^{k}$. Значит, $r\left(f^{k}, \mu, n, \epsilon, \delta\right) \leqslant$ $r(f, \mu, k n, \epsilon, \delta)$, и поэтому $h\left(f^{k}, \mu\right) \leqslant k \cdot h(f, \mu)$.

Замечание 3.3. В однозначном случае имеем тождество $h\left(f^{k}, \mu\right)=k \cdot h(f, \mu)$. Но это тождество отсутствует в настоящем многозначном случае, потому что отображения $d_{n}$ в $(1)$ являются лишь полуметриками, как показано в примере 2.2 .

Нам понадобится следующая лемма. Для любого подмножества $A \subset X$ используем обозначение $B_{\epsilon}[A, \epsilon]=\bigcup_{x \in A} B[x, \epsilon]$.

Лемма 3.4. Пусть $f$ - полунепрерывное сверху замкнутозначное отображение компактного метрического пространства $X$. Если $\left(a^{n}\right) u\left(b^{n}\right)$-последовательности, для которых $a^{n} \rightarrow a$, $b^{n} \rightarrow b u a^{n} \in f\left(b^{n}\right)$ при всех $n \in \mathbb{N}$, то $a \in f(b)$.

Доказательство. Зафиксируем $\epsilon>0$. Из полунепрерывности сверху следует существование такого $N>0$, что $f\left(b^{n}\right) \subset B[f(b), \epsilon]$ при всех $n \geqslant N$. Из $a^{n} \in f\left(b^{n}\right)$ следует, что $a^{n} \in B[f(b), \epsilon]$ для всех $n \geqslant N$. Из этого получаем такую последовательность $x_{n} \in f(b)$, что $d\left(a^{n}, x_{n}\right) \leqslant \epsilon$ при всех $n \geqslant N$. При необходимости переходя к подпоследовательности, можно предположить, что $x^{n} \rightarrow x$ для некоторого $x \in X$. Имеем $x \in f(b)$ (так как $f(b)$ замкнуто) и $d(a, x) \leqslant \epsilon$. Это означает $a \in B[f(b), \epsilon]$. Так как $\epsilon$ произвольно и $f(b)$ замкнуто, получаем $a \in f(b)$.

Лемма 3.5. Если $f$-полунепрерывное сверху замкнутозначное отображение компактного метрического пространства $X$, то $B_{k}[x, \epsilon]$ замкнуто при любых $k \in \mathbb{N}^{+}, \epsilon>0$ u $x \in X$. 
Доказательство. Зафиксируем такую последовательность $y^{n} \in B_{k}[x, \delta]$, что $y^{n} \rightarrow y$ при некотором $y \in X$. Необходимо доказать $y \in B_{k}[x, \epsilon]$.

По определению при всех $n$ существуют такие последовательности $\left(y_{i}^{n}\right)_{i=0}^{k}$ и $\left(x_{i}^{n}\right)_{i=0}^{k}$, что $y_{0}^{n}=y^{n}, x_{0}^{n}=x$,

$$
y_{i+1}^{n} \in f\left(y_{i}^{n}\right), \quad x_{i+1}^{n} \in f\left(x_{i}^{n}\right), \quad d\left(y_{i}^{n}, x_{i}^{n}\right) \leqslant \epsilon \quad \forall 0 \leqslant i \leqslant k-1 .
$$

Очевидно, последовательности $\left(y_{1}^{n}\right)$ и $\left(x_{1}^{n}\right)$ при $n \in \mathbb{N}$ удовлетворяют условиям

$$
y_{1}^{n} \in f\left(y^{n}\right), \quad x_{1}^{n} \in f(x), \quad d\left(y_{1}^{n}, x_{1}^{n}\right) \leqslant \epsilon \quad \forall n \in \mathbb{N} .
$$

При необходимости переходя к подпоследовательности, можно считать, что $y_{1}^{n} \rightarrow y_{1}, x_{1}^{n} \rightarrow x_{1}$ при $n \rightarrow \infty$. Применяя лемму 3.4 , получим

$$
y_{1} \in f(y), \quad x_{1} \in f(x), \quad d\left(y_{1}, x_{1}\right) \leqslant \epsilon .
$$

Далее рассмотрим последовательности $\left(y_{2}^{n}\right)$ и $\left(x_{2}^{n}\right)$ при $n \in \mathbb{N}$. Они удовлетворяют условиям

$$
y_{2}^{n} \in f\left(y_{1}^{n}\right), \quad x_{2}^{n} \in f\left(x_{2}^{n}\right), \quad d\left(y_{2}^{n}, x_{2}^{n}\right) \leqslant \epsilon \quad \forall n \in \mathbb{N} .
$$

При необходимости переходя к подпоследовательности, можно считать, что $y_{2}^{n} \rightarrow y_{2}$ и $x_{2}^{n} \rightarrow x_{2}$. Тогда из леммы 3.4 следует, что

$$
y_{2} \in f\left(y_{1}\right), \quad x_{2} \in f\left(x_{1}\right), \quad d\left(y_{2}, x_{2}\right) \leqslant \epsilon .
$$

Повторяя процесс, получаем последовательности $\left(y_{i}\right)_{i=0}^{k}$ и $\left(x_{i}\right)_{i=0}^{k}$, удовлетворяющие условиям $y_{0}=y, x_{0}=x$,

$$
y_{i+1} \in f\left(y_{i}\right), \quad x_{i+1} \in f\left(x_{i}\right), \quad d\left(y_{i}, x_{i}\right) \leqslant \epsilon \quad \forall 0 \leqslant i \leqslant k-1 .
$$

Из этого следует, что $y \in B_{k}[x, \epsilon]$.

Лемма 3.6. Если $f$-полунепрерывное сверху замкнутозначное отображение компактного метрического пространства $X$, то

$$
h(f, \mu) \geqslant \lim _{\epsilon \rightarrow 0} \limsup _{n \rightarrow \infty}-\frac{1}{n} \log \sup _{x \in X} \mu\left(B_{n}[x, \epsilon]\right),
$$

для любой вероятностной борелевской меры $\mu$ пространства $X$.

Доказательство. Заметим, что по лемме 3.5 все шары $B_{n}[x, \epsilon]$ замкнуты (следовательно, измеримы). Докажем неравенство

$$
r(n, \epsilon, \delta) \geqslant \frac{1-\delta}{\sup _{x \in X} \mu\left(B_{n}[x, \epsilon]\right)} \quad \forall n \in \mathbb{N}^{+}, \forall \epsilon>0, \forall 0<\delta<1 .
$$

Если $r(n, \epsilon, \delta)=\infty$, то доказывать нечего. В противном случае выбираем такое конечное $(n, \epsilon, \delta)$ порождающее множество $E$ для $\mu$, что $\operatorname{card}(E)=r(n, \epsilon, \delta)$. Так как $B_{n}[x, \epsilon]$ измеримо и $E$ конечно, заключаем, что $\bigcup_{x \in E} B_{n}[x, \epsilon]$ также измеримо. Так как $E$ является $(n, \epsilon, \delta)$-порождающим для $\mu$, имеем

Тогда

$$
\mu\left(\bigcup_{x \in E} B_{n}[x, \epsilon]\right)=1-\mu\left(X \backslash \bigcup_{x \in E} B_{n}[x, \epsilon]\right) \geqslant 1-\delta .
$$

$$
\begin{aligned}
r(n, \epsilon, \delta) \cdot \sup _{x \in X} \mu\left(B_{n}[x, \epsilon]\right)=\operatorname{card}(E) \cdot \sup _{x \in X} \mu\left(B_{n}[x, \epsilon]\right) \geqslant \\
\quad \geqslant \sum_{x \in E} \mu\left(B_{n}[x, \epsilon]\right) \geqslant \mu\left(\bigcup_{x \in E} B_{n}[x, \epsilon]\right) \geqslant 1-\delta,
\end{aligned}
$$

что доказывает (5). Переходя к верхнему пределу в (5), получаем

$$
h(f, \mu, \epsilon, \delta) \geqslant \limsup _{n \rightarrow \infty}-\frac{1}{n} \log \sup _{x \in X} \mu\left(B_{n}[x, \epsilon]\right) \quad \forall 0<\delta<1, \epsilon>0,
$$

что доказывает (4). 
Лемма 3.7. Пусть $f$-многозначное отображение метрического пространства $X$. Если $\epsilon_{0}>0 u \lambda>0$ таковы, что $\operatorname{dist}(f(x), f(y)) \geqslant \lambda d(x, y)$ при всех $x, y \in X$, удовлетворяющих условию $d(x, y) \leqslant \epsilon_{0}$, mо

$$
B_{n}[x, \epsilon] \subset B\left[x, \lambda^{1-n} \epsilon\right] \quad \forall n \in \mathbb{N}^{+}, \forall 0<\epsilon<\epsilon_{0}, \forall x \in X .
$$

Доказательство. Зафиксируем $n \in \mathbb{N}^{+}, 0<\epsilon<\epsilon_{0}$ и $x \in X$. Возьмем $y \in B_{n}[x, \epsilon]$. Тогда существуют такие последовательности $\left(x_{i}\right)_{i=0}^{n}$ и $\left(y_{i}\right)_{i=0}^{n}$, что $x_{0}=x, y_{0}=y, x_{i+1} \in f\left(x_{i}\right), y_{i+1} \in f\left(y_{i}\right)$ и $d\left(x_{i}, y_{i}\right) \leqslant \epsilon$ при $0 \leqslant i \leqslant n-1$.

Так как $\epsilon<\epsilon_{0}$, имеем $d(x, y) \leqslant \epsilon_{0}$ и также $\operatorname{dist}(f(x), f(y)) \geqslant \lambda d(x, y)$. Тогда $d\left(x_{1}, y_{1}\right) \geqslant \lambda d(x, y)$. Так как $\epsilon<\epsilon_{0}$, имеем $d\left(x_{1}, y_{1}\right) \leqslant \epsilon_{0}$ и также $\operatorname{dist}\left(f\left(x_{1}\right), f\left(y_{1}\right)\right) \geqslant \lambda d\left(x_{1}, y_{1}\right)$. Тогда

$$
d\left(x_{2}, y_{2}\right) \geqslant \lambda d\left(x_{1}, y_{1}\right) \geqslant \lambda^{2} d(x, y) .
$$

Так как $\epsilon<\epsilon_{0}$, имеем $d\left(x_{2}, y_{2}\right) \leqslant \epsilon_{0}$ и также $\operatorname{dist}\left(f\left(x_{2}\right), f\left(y_{2}\right)\right) \geqslant \lambda d\left(x_{2}, y_{2}\right)$. Тогда

$$
d\left(x_{3}, y_{3}\right) \geqslant \lambda d\left(x_{2}, y_{2}\right) \geqslant \lambda^{3} d(x, y) .
$$

Повторяя процесс, приходим к неравенству $d\left(x_{n-1}, y_{n-1}\right) \geqslant \lambda^{n-1} d(x, y)$. Тогда

$$
d(x, y) \leqslant \lambda^{1-n} d\left(x_{n-1}, y_{n-1}\right) \leqslant \lambda^{1-n} \epsilon ;
$$

таким образом, $y \in B\left[x, \lambda^{1-n} \epsilon\right]$.

Доказательство теоремы 1.4. Пусть $f$ - полунепрерывное сверху замкнутозначное отображение компактного метрического пространства $X$. Пусть $\mu$ - вероятностная борелевская мера пространства $X$ с нижней размерностью $\underline{d}(\mu)$. Сначала докажем неравенство

$$
h(f, \mu) \geqslant \underline{d}(\mu) \cdot \log E C(f) .
$$

Если $\underline{d}(\mu)=0$ либо $E C(f) \leqslant 1$, то доказывать нечего. Тогда считаем, что $\underline{d}(\mu)>0$ и $E C(f)>1$.

Зафиксируем $0<\alpha<\underline{d}(\mu)$. Очевидно, $\underline{d}(\mu)-\alpha<\underline{d}(\mu)$. Тогда из определения $\underline{d}(\mu)$ следует (см., например, (3)), что существует такое $0<\beta<1$, что

$$
\frac{\log \mu(B[x, r])}{\log r}>\underline{d}(\mu)-\alpha \quad \forall 0<r<\beta, \forall x \in X .
$$

Возьмем $0<r<\beta$ и $x \in X$. Так как $\beta<1, r<1$, то $\log r<0$. Таким образом,

$$
\log \mu(B[x, r])<(\underline{d}(\mu)-\alpha) \log r \quad \text { либо } \quad \mu(B[x, r])<r^{\underline{d}}(\mu)-\alpha
$$

при всех $x \in X$. Отсюда получаем

$$
\sup _{x \in X} \mu(B[x, r]) \leqslant r^{\underline{d}(\mu)-\alpha} \quad \forall 0<r<\beta .
$$

Зафиксируем также $1<\rho^{*}<E C(f)$. Из определений следует, что существует такое $\epsilon_{0}>0$, что $\operatorname{dist}(f(x), f(y)) \geqslant \rho^{*} d(x, y)$, когда $x, y \in X$ удовлетворяют $d(x, y) \geqslant \epsilon_{0}$. Так как $\rho^{*}>1$, то можно выбрать такое $N \in \mathbb{N}$, что

$$
\left(\rho^{*}\right)^{1-n} \epsilon<\beta \quad \forall n \geqslant N, \forall 0<\epsilon<\epsilon_{0} .
$$

Заменяя $r=\left(\rho^{*}\right)^{1-n} \epsilon$ в (8), получим

$$
\sup _{x \in X} \mu\left(B\left[x,\left(\rho^{*}\right)^{1-n} \epsilon\right]\right) \leqslant\left(\left(\rho^{*}\right)^{1-n} \epsilon\right)^{\underline{d}(\mu)-\alpha} \quad \forall n \geqslant N, \forall 0<\epsilon<\epsilon_{0} .
$$

С другой стороны, комбинируя леммы 3.6 и 3.7 , получаем нижнюю оценку

$$
h(f, \mu) \geqslant \lim _{\epsilon \rightarrow 0} \limsup _{n \rightarrow \infty}-\frac{1}{n} \log \sup _{x \in X} \mu\left(B\left[x,\left(\rho^{*}\right)^{1-n} \epsilon\right]\right) .
$$


Тогда

$$
\begin{aligned}
h(f, \mu) \geqslant \lim _{\epsilon \rightarrow 0} \limsup _{n \rightarrow \infty}-\frac{1}{n} \log \left(\left(\rho^{*}\right)^{1-n} \epsilon\right)^{\underline{d}(\mu)-\alpha}= & \\
=\lim _{\epsilon \rightarrow 0} \limsup _{n \rightarrow \infty}\left(\frac{n-1}{n}(\underline{d}(\mu)-\alpha) \log \rho^{*}-\frac{1}{n}(\underline{d}(\mu)-\alpha) \cdot \log \epsilon\right) & =(\underline{d}(\mu)-\alpha) \log \rho^{*} .
\end{aligned}
$$

Полагая $\rho^{*} \rightarrow E C(f)$ и $\alpha \rightarrow 0^{+}$, получаем (7).

В заключение заметим, что $f^{k}$ полунепрерывно сверху при всех $k \in \mathbb{N}^{+}$, так как $f$ полунепрерывно сверху. Более того, так как $f$ замкнутозначно, можно доказать, как в лемме 3.5 , что $f^{k}$ также замкнутозначно. Следовательно, в (7) можно заменить $f$ на $f^{k}$, в результате чего получим

$$
h\left(f^{k}, \mu\right) \geqslant \underline{d}(\mu) \cdot E C\left(f^{k}\right) \quad \forall k \in \mathbb{N}^{+} .
$$

Тогда из леммы 3.2 следует $h(f, \mu) \geqslant \underline{d}(\mu) \cdot \frac{1}{k} \log E C\left(f^{k}\right)$ для всех $k \in \mathbb{N}^{+}$, а также

$$
h(f, \mu) \geqslant \underline{d}(\mu) \cdot \lim _{k \rightarrow \infty} \frac{1}{k} \log E C\left(f^{k}\right)=\underline{d}(\mu) \cdot \xi(f),
$$

что завершает доказательство.

\section{СПИСОК ЛИТЕРАТУРЫ}

1. Динабург Е. И. Соотношение между топологической энтропией и метрической энтропией// Докл. АН CCCP. - 1970. - 190, № 1. - С. 19-22.

2. Колмогоров A. Н. Новый метрический инвариант транзитивных динамических систем и автоморфизмов пространств Лебега// Тр. Мат. ин-та им. В. А. Стеклова. - 1985. - 169. - С. 94-98.

3. Синай Я. Г. О понятии энтропии динамической системы// Докл. АН СССР. - 1959. - 124. - С. $768-$ 771.

4. Хинчин А. Я. Об основных теоремах теории информации// Усп. мат наук. - 1956. - 11, № 1 (67). C. $17-75$.

5. Adler R. L., Konheim A. G., McAndrew M. H. Topological entropy// Trans. Am. Math. Soc. - 1965. 114. - C. 309-319.

6. Akin E. The General Topology of Dynamical Systems. - Providence, Rhode Island: Am. Math. Soc., 1993.

7. Aubin J.-P., Cellina A. Differential Inclusions. Set-Valued Maps and Viability Theory. - Berlin: SpringerVerlag, 1984.

8. Aubin J.-P., Frankowska H. Set-Valued Analysis. - Boston, Massachusetts: Birkhäuser, 2009.

9. Aubin J.-P., Frankowska H., Lasota A. Poincaré's recurrence theorem for set-valued dynamical systems// Ann. Polon. Math. - 1991. - 54, № 1. - C. 85-91.

10. Barreira L. Dimension and Recurrence in Hyperbolic Dynamics. - Basel: Birkhäuser, 2008.

11. Barreira L., Pesin Y., Schmeling J. Dimension and product structure of hyperbolic measures// Ann. Math. (2) - 1999. - 149, № 3. - C. 755-783.

12. Blumenthal L. M. A new concept in distance geometry with applications to spherical subsets// Bull. Am. Math. Soc. - 1941. - 47. - C. 435-443.

13. Boltzmann L. Über die beziehung dem zweiten Haubtsatze der mechanischen Wärmetheorie und der Wahrscheinlichkeitsrechnung respektive den Sätzen über das Wärmegleichgewicht// Wiener Berichte. 1877. - 76. - C. 373-435.

14. Bowen R. Entropy for group endomorphisms and homogeneous spaces// Trans. Am. Math. Soc. - 1971. 153. - C. 401-414.

15. Burachik R. S., Iusem A. N. Set-Valued Mappings and Enlargements of Monotone Operators. - New York: Springer-Verlag, 2008.

16. Carrasco-Olivera D., Metzger R., Morales C. A. Topological entropy for set-valued maps// Discr. Contin. Dyn. Syst. Ser. B - 2015. - 20, № 10. - C. 3461-3474.

17. Cheng W.-C., Zhao Y., Cao Y. Pressures for asymptotically sub-additive potentials under a mistake function// Discr. Contin. Dyn. Syst. - 2012. - 32, № 2. - C. 487-497.

18. Clausius $R$. Über verschiedene für die Anwendung bequeme Formen der Hauptgleichungen der mechanischen Wärmetheorie// Ann. Phys.. - 125. - C. 353-400. 
19. Clausius R. The Mechanical Theory of Heat with Its Applications to the Steam Engine and to Physical Properties of Bodies. - London: John van Voorst, 1867.

20. Dai X., Zhou Z., Geng X. Some relations between Hausdorff dimensions and entropies// Sci. China Ser. A. - 1998. - 41, № 10. - C. 1068-1075.

21. Downarowicz T. Entropy in Dynamical Systems. - Cambridge: Cambridge Univ. Press, 2011.

22. Fathi A. Expansiveness, hyperbolicity and Hausdorff dimension// Commun. Math. Phys. - 1989. - 126, № 2. - C. 249-262.

23. Gillam B. E. A new set of postulates for Euclidean geometry// 42 - 1940. - C. 869-899.

24. Hirsch M. Asymptotic phase, shadowing and reaction-diffusion systems// Lect. Notes Pure Appl. Math. 1994. - 152. - C. 87-99.

25. Katok A. Fifty years of entropy in dynamics: 1958-2007// J. Mod. Dyn. - 2007. - 1, № 4. - C. 545-596.

26. Katok A. Lyapunov exponents, entropy and periodic orbits for diffeomorphisms// Inst. Hautes Études Sci. Publ. Math. - 1980. - 51. - C. 137-173.

27. Maschler M., Peleg B. Stable sets and stable points of set-valued dynamic systems with applications to game theory// SIAM J. Control Optim. - 1976. - 14, № 6. - C. 985-995.

28. Mañé R. Expansive homeomorphisms and topological dimension// Trans. Am. Math. Soc. - $1979 .-252$. - C. 313-319.

29. McMillan B. The basic theorems of information theory// Ann. Math. Stat. - 1953. - 24. - C. $196-219$.

30. Reddy W. L. Expanding maps on compact metric spaces// Topol. Appl. - 1982. - 13, № 3. - C. $327-334$.

31. Rockafellar R. T. Convex Analysis. - Princeton, New Jersey: Princeton Univ. Press, 1997.

32. Schreiber S. Expansion rates and Lyapunov exponents// Discr. Contin. Dynam. Syst. — 1997. — 3, № 3. C. 433-438.

33. Shannon C. E. A mathematical theory of communication// Bell System Tech. J. - 1948. - 27. - C. 379423, 623-656.

34. Sinai Y. Kolmogorov-Sinai entropy// Scholarpedia. - 2009. - 4, № 3. - C. 2034.

35. Tarafdar E., Watson P., Yuan X.-Z. Poincare's recurrence theorems for set-valued dynamical systems// Appl. Math. Lett. - 1997. - 10, № 6. - C. 37-44.

36. Tarafdar E., Yuan X.-Z. The set-valued dynamic system and its applications to Pareto optima// Acta Appl. Math. - 1997. - 46, № 1. - C. 93-106.

37. von Neumann J. Mathematische Grundlagen der Quantenmechanik. - Berlin-New York: Springer-Verlag, 1968 (in German).

38. Walters P. An Introduction to Ergodic Theory. - New York-Berlin: Springer-Verlag, 1982.

39. Williams R. K. A note on expansive mappings// Proc. Am. Math. Soc. - 1969. - 22. - C. $145-147$.

Carrasco-Olivera D.

Университет Био-Био, Консепсьон, Чили

E-mail: dcarrasc@ubiobio.cl

Metzger R.

Национальный инженерный университет, Лима, Перу

E-mail: metzger@imca.edu.pe

Morales C. A.

Федеральный университет Рио-де-Жанейро, Рио-де-Жанейро, Бразилия

E-mail: morales@impa.br 